\title{
Design of Reconfigurable Micro fluidic Met surface with Different Semiconductor Alloys
}

\author{
A. AlGhamdi ${ }^{1}$, J. Yousaf ${ }^{2}$, A. Yahyaoui ${ }^{3}$, N. Sobahi ${ }^{4}$, H. Rmili ${ }^{5}$ \\ 1,5Electrical and Computer Engineering Department, Faculty of Engineering, King Abdulaziz University, P.O. Box 80204, Jeddah \\ 21589, Saudi Arabia. \\ ${ }^{2}$ Department of Electrical, Computer, and Biomedical Engineering, Abu Dhabi University, United Arab Emirates. \\ ${ }^{3,4}$ Electrical and Electronic Engineering Department, College of Engineering, University of Jeddah, Saudi Arabia. \\ ${ }^{4}$ Communications Systems Laboratory (SysCom), National Engineering School of Tunis (ENIT), University of Tunis El Manar \\ (UTM), BP 37, Belvédère 1002 Tunis, Tunisia.
}

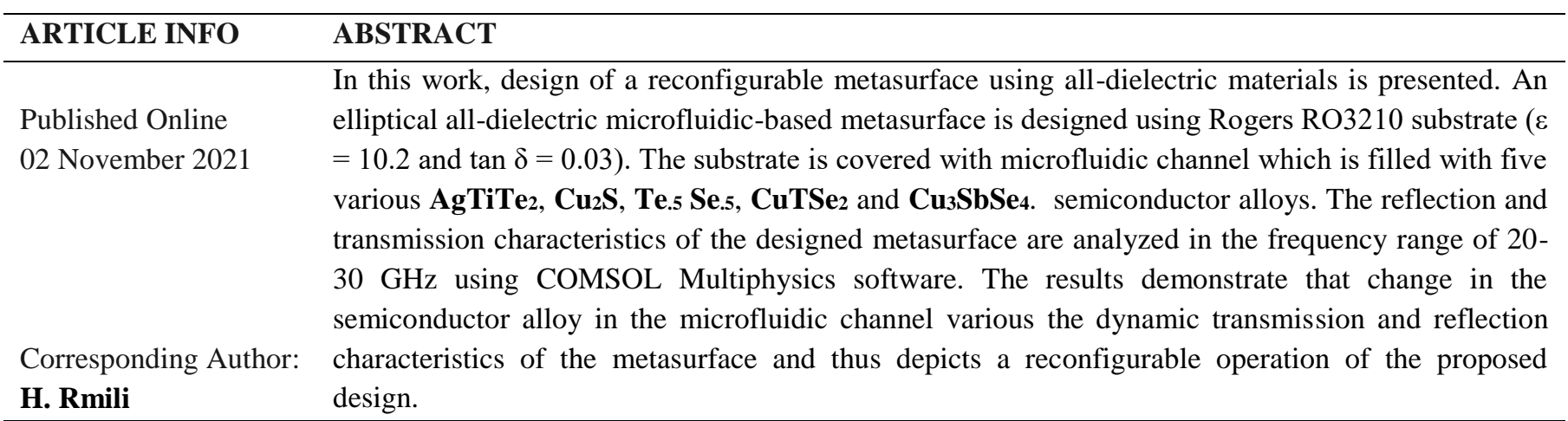

KEYWORDS: All-dielectric, metasurface, reconfigurable, microfluidic, transmission coefficient

\section{INTRODUCTION}

Metasurfaces are made of the periodic structure of subwavelength elements. The arrangement of sub-wavelength elements in a periodic fashion alters the magnitude, phase, and polarization of the illuminating EM waves. This leads to the design of various polarization converters and filters [14], optical networks [3, 5], reconfigurable communication systems $[1,3,6,7]$, electronic beam steering, bandwidth, and gain enhancement of antennas [3, 7, 8] using metasurfaces.

Reconfigurable nature of the metasurface is achieved by varying the impedance characteristics of elementary units of metasurfaces by using internal or external stimulus. The wavefronts of the EM wavs are altered using either passive or active components to depicts the switching operation of the reconfigurable metasurface. This could be achieved by adding various active components such as diodes, liquid crystals and MEMS based switches in the structure [1, 2, 6, 7, 9-11]). Researchers also demonstrated the variations in impedance characteristics by using alloys and fluid metals as in [12], [13] [14].

This work presents an extensive analysis of the reconfigurable nature of the designed all-dielectric elliptical metasurface. The elementary units of designed metasurface have elliptical dielectric resonator (EDR) shape. The Rogers RO3210 substrate $(\varepsilon=10.2$ and $\tan \delta=0.03$ ) is used for the construction of structure as depicted in Figure 1 . A polydimethylsiloxane (PDMS) channel covering the wings of EDR is placed on the top of the structure. This microfluid

channel is filled with five different semiconductor alloys. The employed semiconductor alloys in the presented study are $\mathrm{AgTiTe}_{2}, \mathrm{Cu}_{2} \mathrm{~S}, \mathrm{Te}_{.5} \mathrm{Se}_{.5}, \mathrm{CuTSe}_{2}$ and $\mathrm{Cu}_{3} \mathrm{SbSe}_{4}$. For each case of semiconductor alloy, the transmission and reflection parameters of the metasurface are obtained in the frequency range of 20 to $30 \mathrm{GHz}$. The employed EM simulation tool for the subject analysis is COMSOL Multiphysics software. The results depict that the wideband transmission and reflection characteristics of the realized metasurface varies with the change in the electrical properties of the used alloys. The details of realized design and results are given in the following sections. 


\section{METASURFACE DESIGN}

A 3D model of dielectric (Rogers RO3210) with relative permittivity 10.2 , loss tangent 0.003 , and thickness $g$, (shown in Figure 1), was employed as a building block of metasurface in this study. the metasurface is designed using COMSOL 5.4 Multiphysics software. On top of the structure, along the $\mathrm{x}$-axis there is a microfluidic channel with the size of $100 \times 100 \mu \mathrm{m}$ which will fill with with different alloys and covered by polydimethylsiloxane (PDMS). The PDMS is placed on top of the wings only with width (e) and thickness of (d) as illustrated in Figure 1. The minor (along $x$ ) and major radii (along $y$ ) of the elliptical dielectric resonator (EDR) are denoted as $a$ and $b$, respectively, whereas its ellipticity is $\tau=b / a$. The infinite all-connected twodimensional EDR structure is shown in Figure 1(b). The size of the unit cell of the dielectric resonator is $L x \times L y \times L z$. In this regard, $L x=9.9 \mathrm{~mm}(L x<\lambda), L y=9.9 \mathrm{~mm}(L y<\lambda)$, and $L z=16 \lambda$, where $\lambda=10 \mathrm{~mm}$ is the wavelength associated with the upper-frequency limit of $30 \mathrm{GHz}$ in the analysed frequency range.

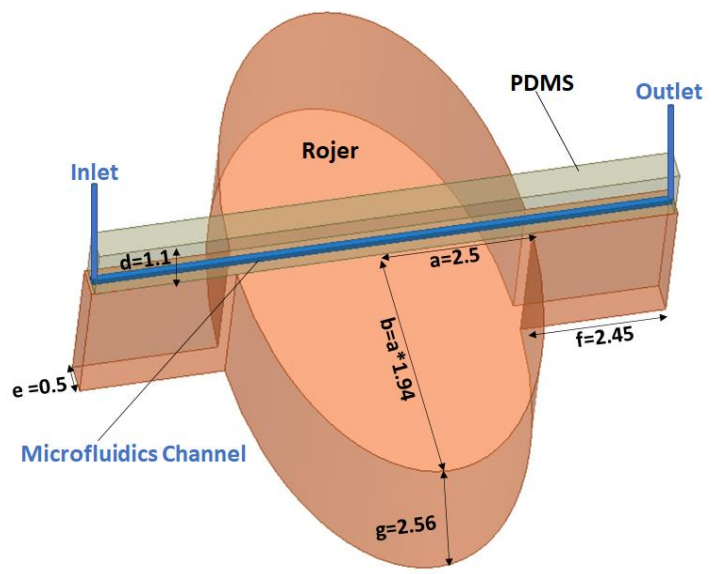

(a)

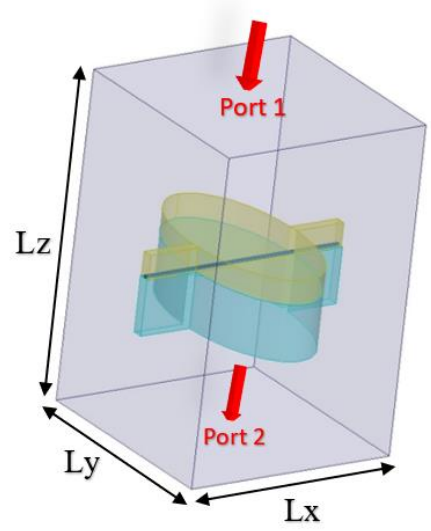

(b)

Figure 1: Schematic of the proposed metasurface: (a) Model configuration (All units in mm), and (b) COMSOL model for the unit cell (port1\&2 are periodic port).

The longitudinal dimension $L z$ is great compared to the wavelength $\lambda(L z=160 \mathrm{~mm})$. The resonators are connected along $x$-direction with a thin connection length of $\mathrm{f}=(L x / 2)$ $-b$ and width $\mathrm{g}=2.56 \mathrm{~mm}$. The structure is excited with normal incidence with a periodic port along $Z$-direction.

\section{RESULTS AND DISCUSSION}

The results of the metasurface when we inject the channel with alloy $\mathrm{Cu}_{3} \mathrm{SbSe}_{4}$ are shown in Figure 2. It starts with high transmission in the band of $20-21.05 \mathrm{GHz}$ and the changes to low transmission ( $\mathrm{S} 21 \leq-10 \mathrm{~dB}$ ) in the spectrum of $21.7 \mathrm{GHz}$ to $22.45 \mathrm{GHz}$. Then the behavior again changes to relatively higher transmission till around $26 \mathrm{GHz}$. The lower reflection level $(\mathrm{S} 11 \leq-10 \mathrm{~dB})$ is observed in the region of $27.7 \mathrm{GHz}$ to $29.25 \mathrm{GHz}$. The metasurface is clearly shown the switching between transmission and reflection at different resonators with different bandwidths [15].

The results of the metasurface when we inject the channel with alloy Te.5 Se.5 are shown in Figure 3. It only has a low transmission $(\mathrm{S} 21 \leq-10 \mathrm{~dB})$ in the spectrum of $21.05 \mathrm{GHz}$ to $22.00 \mathrm{GHz}$ and of $28.61 \mathrm{GHz}$ to $28.96 \mathrm{GHz}$ ).

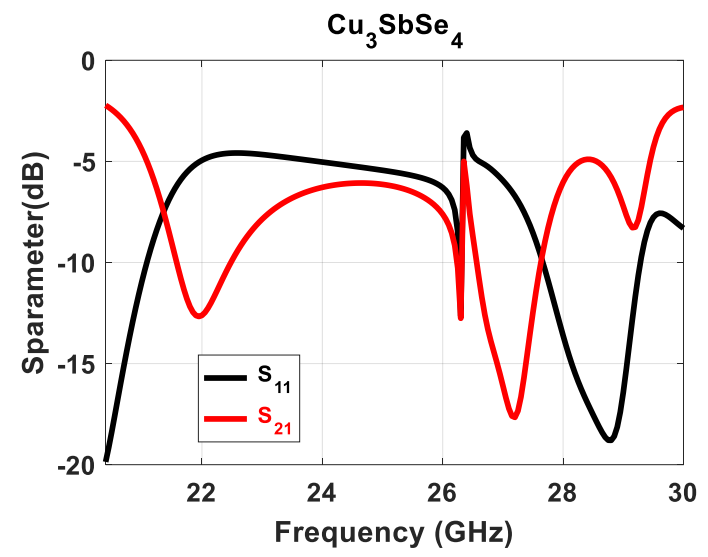

Figure. 2: Simulated results of S-parameters of the model with filling alloy $\mathrm{Cu}_{3} \mathrm{SbSe}_{4}$

The results of the metasurface when we inject the channel with alloy AgTiTe2 are shown in Figure 4. It starts with high transmission in the band of $20-21.75 \mathrm{GHz}$ and the changes to low transmission $(\mathrm{S} 21 \leq-10 \mathrm{~dB})$ in the spectrum of 22.35 $\mathrm{GHz}$ to $23.7 \mathrm{GHz}, 26.5 \mathrm{GHz}$ to $26.95 \mathrm{GHz}$, and $27.4 \mathrm{GHz}$ to $287.95 \mathrm{GHz}[16]$.

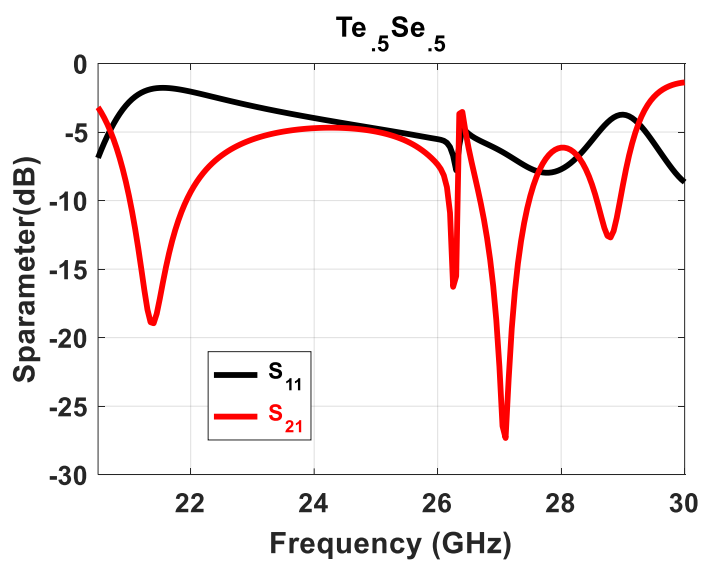

Figure. 3: Simulated results of S-parameters of the model with filling alloy Te.5 Se.5 
The results of the metasurface when we inject the channel with alloy $\mathbf{C u}_{2} \mathbf{S}$ are shown in Figure 5. It starts with high transmission in the band of $20-21.2 \mathrm{GHz}$ and the changes to low transmission $(\mathrm{S} 21 \leq-10 \mathrm{~dB})$ in the spectrum of 21.85 $\mathrm{GHz}$ to $22.55 \mathrm{GHz}$.

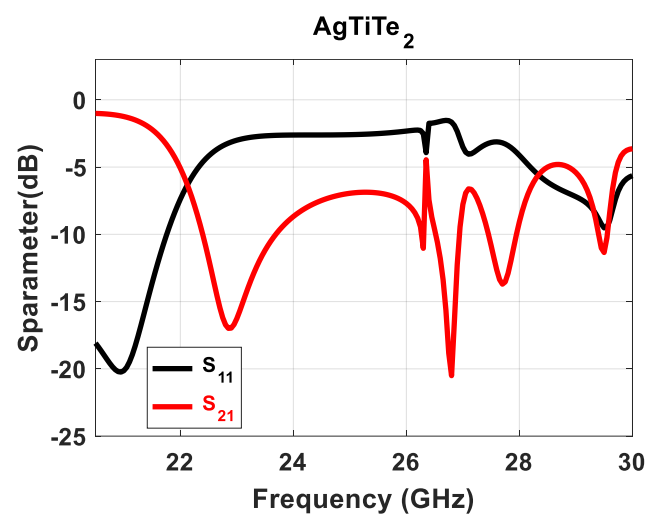

Figure. 4: Simulated results of S-parameters of the model with filling allov AgTiTe2

Then the behavior again changes to relatively higher transmission till around $27.1 \mathrm{GHz}$. The lower reflection level $(\mathrm{S} 11 \leq-10 \mathrm{~dB})$ is observed in the region of $28 \mathrm{GHz}$ to 28.85 GHz. The metasurface is clearly shown the switching between transmission and reflection at different resonators with different bandwidths[17].

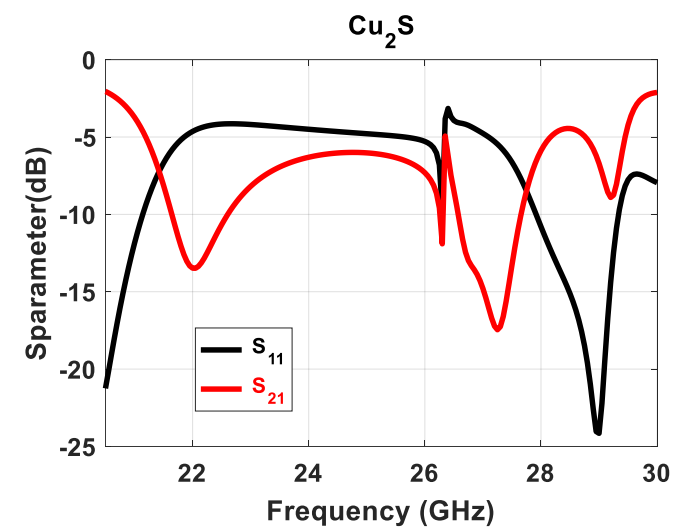

Figure. 5: Simulated results of S-parameters of the model with filling alloy $\mathbf{C u 2} \mathbf{S}$

The results of the metasurface when we inject the channel with alloy CuTSe $_{2}$ are shown in Figure 6. It starts with high transmission in the band of $20-21.50 \mathrm{GHz}$ and the changes to low transmission $(\mathrm{S} 21 \leq-10 \mathrm{~dB})$ in the spectrum of 22.6 $\mathrm{GHz}$ to $23.8 \mathrm{GHz}, 26.5 \mathrm{GHz}$ to $26.9 \mathrm{GHz}$, and $27.6 \mathrm{GHz}$ to 28.1 GHz. The metasurface is clearly shown the switching between transmission and reflection at different resonators with different bandwidths[18].

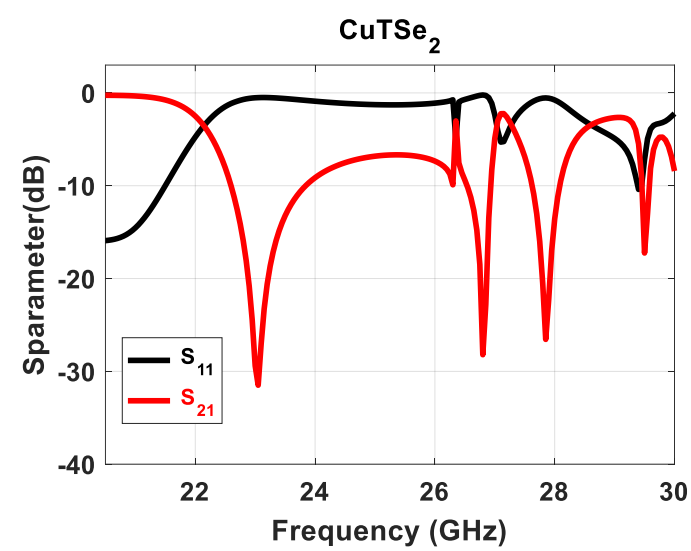

Figure. 6: Simulated results of S-parameters of the model with filling alloy CuTSe2

\section{DISUSSION AND COMPARISON}

This section discusses the comparison of the reconfigurable nature of the realized metasurface.

Figure 7 compares the performance of the Figure 1 metasurface in terms of reflection and transmission parameters with the aforementioned 5 different alloys. We can notice that the variations in the alloys brings a significant change in the reflection and transmission parameters of the metasurface. The change of the alloys brings a change in the electrical properties of the material. The major component of the electrical parameters of a material which play a vital role in the switching operation of the designed metasurface is the conductivity of the material.

The S11 of the $\mathrm{Cu}_{3} \mathrm{SbSe}_{4}, \mathrm{Cu}_{2} \mathrm{~S}, \mathrm{AgTiTe}_{2}$, and $\mathrm{CuTSe}_{2}$ materials starts with dip around $-15 \mathrm{~dB}$ showing good reflection while the waveforms of the Te.5 Se.5 alloy starts at around $-5 \mathrm{~dB}$. This shows poor reflection performance of the Te.5 Se.5 alloy at $20 \mathrm{GHz}$ as compared to other alloys. The reflection parameters of the $\mathbf{C u T S e}_{2}$ approaches to around 0 $\mathrm{dB}$ in the frequency band of 20 to $26 \mathrm{GHz}$. This represents the poor transmission of the metasurface in this frequency band with CuTSe 2 alloy. On the contrary, microfluidic channel with inserted alloys of Te.5 $\mathrm{Se}_{.5}, \mathrm{Cu}_{3} \mathrm{SbSe}_{4}$, and $\mathrm{Cu}_{2} \mathrm{~S}$ brings the $\mathrm{S} 11$ values of the metasurface to around -5 $\mathrm{dB}$ within the same frequency band of 20to $26 \mathrm{GHz}$. Almost identical results are noticed for the metasurfaces with $\mathrm{Cu}_{3} \mathrm{SbSe}_{4}$ and $\mathrm{Cu}_{2} \mathrm{~S}$ alloys which could be due to their similar electrical properties. 


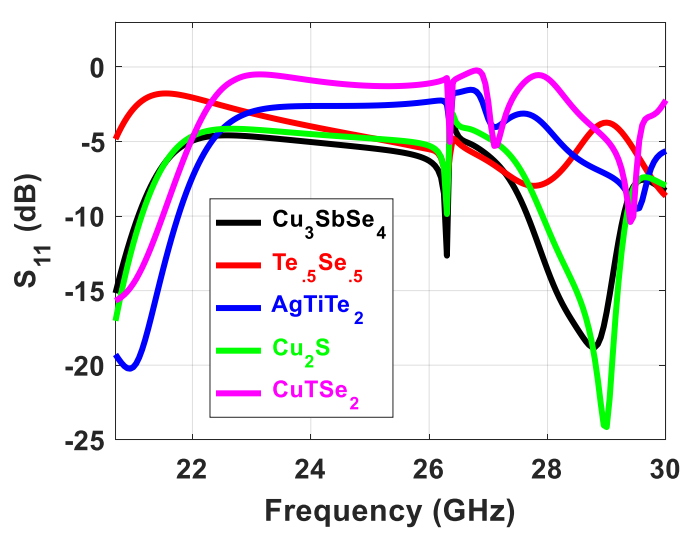

(a)

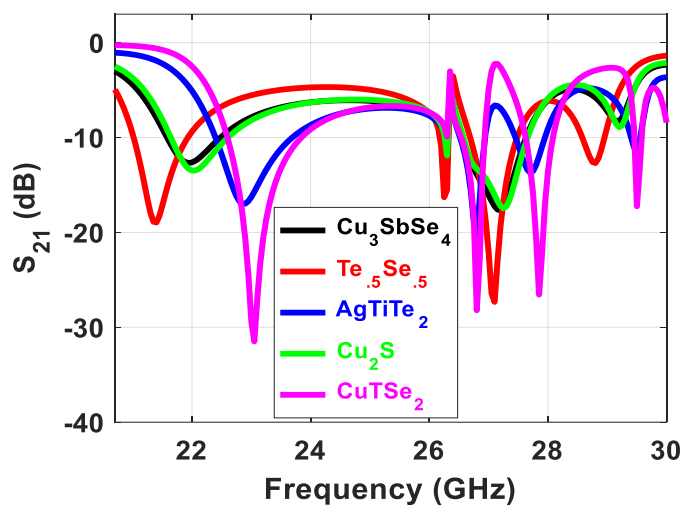

(b)

Figure. 7: Performance comparison of metasurface with different alloys (a) Reflection, (b) Transmission

The variations in the good transmission bands $\mathrm{S} 21>-10 \mathrm{~dB}$ ) can be noticed in Figure 7(b) for all the analyzed cases of liquid alloys. Te.5 Se.5 shows overall superior performance as compared to all other alloys as its transmission curve is above $-10 \mathrm{~dB}$ from 22 to $26 \mathrm{GHz}$. After Te.5 Se.5, Cu3SbSe 4 and $\mathrm{Cu}_{2} \mathrm{~S}$ performed well with higher bandwidth coverage for the $\mathrm{S} 21>-10 \mathrm{~dB}$. The dynamic reconfigurable nature of the realized metasurface can be observed from the varying low and high transmission and reflections bands in Figure 7 curves.

\section{CONCLUSION}

The reconfigurable operation of an all-dielectric elliptical shape metasurface having a microfluidic channel is demonstrated by varying five different semiconductor alloys (AgTiTe2, Cu2S, Te.5-Se.5, CuTSe2 and $\mathrm{Cu}_{3} \mathrm{SbSe}_{4}$ ) in the microfluidic channel. Reconfigurable nature of the realized metasurface is demonstrated through the variations in the high reflection and high transmission bands of the analyzed structure in the frequency range of 20 to $30 \mathrm{GHz}$. The change of conductivity of the semiconductor alloy in the inserted microfluidic channel varies the impedance properties of the EDR units of realized metasurface which changes the transmission and reflection properties of the proposed metasurface design. The proposed design strategy of varying electrical properties of microfluidic alloys could be used for the realization of a low-cost switchable metasurfaces.

\section{ACKNOWLEDGMENT}

This project was funded by the Deanship of Scientific Research (DSR), King Abdulaziz University, Jeddah, Saudi Arabia under grant No. (KEP-PhD-13-135-42). The authors, therefore, acknowledge with thanks DSR technical and financial support.

\section{REFERENCES}

1. J. Wang, R. Yang, R. Ma, J. Tian, and W. Zhang, "Reconfigurable Multifunctional Metasurface for Broadband Polarization Conversion and Perfect Absorption," IEEE Access, vol. 8, pp. 105815105823, 2020.

2. A. Pitilakis et al., "A multi-functional reconfigurable metasurface: Electromagnetic design accounting for fabrication aspects," IEEE Transactions on Antennas and Propagation, pp. 1-1, 2020.

3. Q. He, S. Sun, and L. Zhou, "Tunable/ Reconfigurable Metasurfaces: Physics and Applications," Research, vol. 2019, p. 1849272 , 2019/07/07 2019.

4. A. Yahyaoui and H. Rmili, "Chiral All-Dielectric Metasurface Based on Elliptic Resonators with Circular Dichroism Behavior," International Journal of Antennas and Propagation, vol. 2018, p. 6352418, 2018/04/19 2018.

5. M. Y. Shalaginov et al., "Reconfigurable Alldielectric Metasurfaces based on Optical Phase change Materials: Design Approaches," in 2020 International Applied Computational Electromagnetics Society Symposium (ACES), 2020, pp. 1-2.

6. K. V. Mishra, J. A. Hodge, and A. I. Zaghloul, "Reconfigurable Metasurfaces for Radar and Communications Systems," in 2019 URSI AsiaPacific Radio Science Conference (AP-RASC), 2019, pp. 1-4.

7. S. Zahra et al., "Electromagnetic Metasurfaces and Reconfigurable Metasurfaces: A Review," (in English), Review vol. 8, no. 615, 2021-January-14 2021.

8. S. Foo, "Liquid-crystal reconfigurable metasurface reflectors," in 2017 IEEE International Symposium on Antennas and Propagation \& USNC/URSI National Radio Science Meeting, 2017, pp. 20692070.

9. G. Liu, H. Liu, J. Han, Y. Mu, and L. Li, "Reconfigurable metasurface with polarizationindependent manipulation for reflection and transmission wavefronts," Journal of Physics D: 
Applied Physics, vol. 53, no. 4, p. 045107, 2019/11/18 2019.

10. Y. Li, Y. Wang, and Q. Cao, "Design of a Multifunctional Reconfigurable Metasurface for Polarization and Propagation Manipulation," IEEE Access, vol. 7, pp. 129183-129191, 2019.

11. S. Sun, W. Jiang, S. Gong, and T. Hong, "Reconfigurable Linear-to-Linear Polarization Conversion Metasurface Based on PIN Diodes," IEEE Antennas and Wireless Propagation Letters, vol. 17, no. 9, pp. 1722-1726, 2018.

12. K. Ling, H. K. Kim, M. Yoo, and S. Lim, "Frequency-Switchable Metamaterial Absorber Injecting Eutectic Gallium-Indium (EGaIn) Liquid Metal Alloy," Sensors, vol. 15, no. 11, 2015.

13. H. K. Kim, D. Lee, and S. Lim, "WidebandSwitchable Metamaterial Absorber Using Injected Liquid Metal," Scientific Reports, vol. 6, no. 1, p. 31823, 2016/08/22 2016.

14. P. C. Wu et al., "Broadband Wide-Angle Multifunctional Polarization Converter via LiquidMetal-Based Metasurface," Advanced Optical Materials, https://doi.org/10.1002/adom.201600938 vol. 5, no. 7, p. 1600938, 2017/04/01 2017.

15. L. Min, Y. Xia, P. Ying, and J. Cui, "Hybrid structure responsible for improved thermoelectric performance of Sn-incorporated $\mathrm{Cu}_{3} \mathrm{SbSe}_{4}$ with a second phase CuSe," Journal of Applied Physics, vol. 127, no. 23, p. 235104, 2020.

16. O. Uemura, K. Suzuki, and T. Satow, "Electrical and Magnetic Properties of the Molten Ag-TlTe System," Berichte der Bunsengesellschaft für physikalische Chemie, vol. 88 , no. 5, pp. 473-478, 1984.

17. A. Barnes and J. Enderby, "The structural and electrical properties of liquid copper selenide," Philosophical Magazine B, vol. 58, no. 5, pp. 497512, 1988 .

18. S. Tomar, R. Gautam, C. NEGI, S. GUPTA, S. BHARDWAJ, and A. VERMA, "AB INITIO STUDIES OF STRUCTURAL, ELECTRONIC, OPTICAL, ELASTIC AND THERMAL PROPERTIES OF COPPER THALLIUMDICHALCOGENIDES (CuTiX 2: $\mathrm{X}=$ $\mathrm{S}, \mathrm{Se}, \mathrm{Te}), "$ Chalcogenide Letters, vol. 16 , no. 1 , 2019. 